\title{
The placebo effect from the biopsychosocial perspective of general medicine: non-effective interventions that are, in fact, effective
}

\begin{abstract}
There are recovery mechanisms of the disease that are more complex than homeostasis. Among these is the placebo effect. They are true social, cultural and psychobiological responses that can significantly modify the overall outcome of the treatment. The effects of placebo and nocebo occur frequently and are clinically significant, but are not recognized despite the comprehensive framework of general medicine. From a biopsychosocial view, a number of factors related to placebo can be differentiated: 1 . The nature and amount of placebo; 2 . The situation and the manner in which the placebo is prescribed in the context of the doctor-patient relationship; 3 . The psychosocial factors of the patient receiving the placebo; 4 . The disease that is treated; 5 . The mode of action of the placebo; 6 . The side effects of placebo; 7. The use of placebo and its ethical aspects; 8. Interaction of specific and non-specific effects; and 9. The healing or symptom relief controversy. General practitioners should be able to recognize these phenomena and master tactics on how to manage these effects to improve the quality of clinical practice. Each treatment in general medicine determines, at the same time, biological, psychological and social effects, these can be specific and contextual (or nonspecific) effects, which interact with each other. General practitioners must manage the factors of the placebo effect as a driver of any active therapeutic intervention to improve the positive effects of the treatment and avoid harmful nocebo effects.
\end{abstract}

Volume 3 Issue I - 2019

\author{
Jose Luis Turabian \\ Specialist in Family and Community Medicine, Health Center \\ Santa Maria de Benquerencia, Regional Health Service of Castilla \\ la Mancha (SESCAM), Toledo, Spain
}

Correspondence: Jose Luis Turabian, Specialist in Family
and Community Medicine, Health Center Santa Maria de
Benquerencia Toledo, Spain, Email jturabianf@hotmail.com

Received: December 31, 2018 | Published: January 28, 2019

Keywords: communication, physician-patient relations, nocebo, placebo, specific and non-specific treatment effects, treatment context

\section{Introduction}

There are natural mechanisms of recovery from the disease that are actually more complex, powerful, and interesting than what Cannon called homeostasis. The body's response to disease is more than homeostasis; it is more a creative adaptation that achieves changes in body and mind. ${ }^{1}$ Among these complex mechanisms, the placebo effect is well known: it has a quantifiable effect on health and is not a medicine, nor does it exert any therapeutic action in itself. Its official study began in the year 1800 when the British doctor John Haygarth made the first systematic study of the healing capacity of methods without therapeutic value. ${ }^{2}$ More than 200 years later, little else we know about the placebo. The name of placebo does not appear in the medical literature until 1832 and its origin is doubtful. ${ }^{3}$ The phenomena of placebo and nocebo surprise and confuse. They are true social, cultural and psychobiological responses that can significantly modify the overall outcome of the treatment. The response to placebo is associated with the expectation of reward and the relief of anticipatory anxiety, while the response of nocebo is related to the lack of reward or positive expectation and the increase of anticipatory anxiety. ${ }^{4}$ The placebo effect has evolved from being considered a nuisance in clinical and pharmacological research to being considered a biological phenomenon worthy of scientific research in its own right. The study of the placebo effect and its negative counterpart, the nocebo effect, is basically the study of the psychosocial context around the treatment and the patient, and plays a crucial role in the therapeutic outcome. ${ }^{5}$ So, placebo is defined as an inert substance that provokes perceived benefits, whereas the term nocebo is used when an inert substance causes harm. ${ }^{6}$

A broader definition of what placebos are and how they act would be: "any therapeutic procedure (or a component of the therapeutic procedure) that is deliberately given to have an effect, or that unknowingly has an effect on the patient's symptoms or illness, but that objectively does not have a specific activity for the treated condition." The placebo effect is defined as the changes produced by the placebos. ${ }^{7}$ And in contrast, the nocebo effect is defined as the worsening of the symptoms induced by any negative attitude of the non-pharmacological therapeutic intervention, both active and inactive therapies. When a patient anticipates a negative effect associated with an intervention, medication or change in medication, he or she may experience an increase in this effect or experience it initially. ${ }^{8}$ It can be said that, in evolutionary terms, nocebo and placebo effects coexist to favour the perceptive mechanisms that anticipate dangerous threats and events (nocebo effects) and promote safety behaviours (placebo effects). ${ }^{9}$ In medical consultations and in research studies the placebo and nocebo effects are difficult to separate from the natural course of the disease or the actual effects of a new medication. Expectations have the potential to relieve many symptoms. Studies of expectations in the field of medicine suggest that the effects of placebo are probably an important aspect of any treatment. The application of research on health-related expectations has the potential to promote wellness, improve health and benefit patient care. ${ }^{10}$

In evidence-based medicine, treatment efficacy can be determined as the difference between the effects of the active study drug and placebo, the latter being a substance that lacks specific action on the disease in question. However, improvements in the conditions of patients under placebo treatment can be substantial and comparable with those of active drug. ${ }^{11}$ Controlled clinical trials neutralize the placebo effect: they are designed specifically to ensure the comparability of the groups (by randomization or pairing), so that only the treatment investigated is different in the groups. However, frequently the placebo effect is more important than the effect of the 
specific drug, but paradoxically we have no information about it. Usually, at least until recently, biomedical researchers disregarded the placebo effect; thus, controlled clinical trials, in their current habitual version (without taking into account psychosocial influences), have the effect of maintaining the anachronism of the biomedical model. ${ }^{12}$ On the other hand, it is necessary to differentiate between genuine placebos and psudoplacebos: the former predominate in clinical studies, while the pseudo-placebos prevail in "alternative" practical therapy. The term pseudoplacebo can also be applied to many procedures in complementary medicine, including homeopathic medicine. ${ }^{11}$ On the other hand, it must be remembered that general medicine / family medicine is defined in biopsychosocial terms (not in biomedical terms exclusively). The biopsychosocial approach identifies the biological, psychological and social factors as elements that are always present and with interrelated influences on health and illness. The application of this theoretical framework involves determining in each patient the specific proportion of the importance of each one of the biological, psychological and social factors in each period of time.

The work skills in this biopsychosocial model are crucial for the general practitioner (GP), who should be able to apply this perspective with a panoramic vision to achieve a complete understanding of the patient and its context. In this way, GPs know that the elements of the patient's personality and their psychosocial and contextual relationships have a great importance in disease and health. ${ }^{13}$ In this paradigm, general medicine does not maintain the mind / body, functional / organic, quantitative / qualitative, biological / psychosocial dualisms. In this way, the study of the placebo effect that is inserted precisely in this biopsychosocial model and in the mind-body interactions, ${ }^{14}$ is a subject that falls squarely, and has great importance, in the field of work of general medicine. In this scenario, it must be distinguished disease from illness. Disease is what a pathologist or physician can observe. It may be a peptic ulcer, seen as a crater in the lining of the stomach or duodenum, or diabetes signified by a high bloodsugar test result. In such cases, the patient's symptoms have a tangible cause, and treatment can be logical and precise. In contrast, illness is what one feels. Illness may accompany a known disease, or not, but the patient feels ill. If the underlying disease is cured by appropriate treatment, the symptoms usually improve. However, what if there is illness without disease? Those suffering from such disorders will be disappointed to discover that there is no specific treatment. Nevertheless, in the situation of illness vs. disease, there are measures that GP can deploy to alleviate the illness. In this context, this article tries to achieve an approach toward systematization and integration of the concept of placebo / nocebo within the practice of general medicine.

\section{Discussion}

From a broad biopsychosocial view in general medicine, a number of factors related to placebo can be differentiated (7): 1) The nature and amount of placebo; 2) The situation and the manner in which the placebo is prescribed in the context of the doctor-patient relationship; 3) The psychosocial factors of the patient receiving the placebo; 4) The disease that is treated; 5) The mode of action of the placebo; 6) The side effects of placebo; 7) The use of placebo and its ethical aspects; 8) Interaction of specific and non-specific effects; and 9) The healing or symptom relief controversy (Table 1).

Table I Factors related to the placebo effect in the biopsychosocial vision of general medicine

\begin{tabular}{ll}
\hline \multicolumn{1}{l}{ Factors related to the placebo effect in the biopsychosocial vision of general medicine } \\
\hline 1 & The nature and amount of placebo \\
2 & The situation and the way in which the placebo is prescribed in the context of the doctor-patient relationship \\
3 & The psychosocial factors of the patient receiving the placebo \\
4 & The disease being treated \\
5 & The mode of action of the placebo \\
6 & Side effects of placebo \\
7 & The use of placebo and its ethical aspects \\
8 & The interaction of specific and non-specific effects \\
9 & Healing or relief of symptoms \\
\hline
\end{tabular}

\section{The nature and amount of placebo}

The metaphors and symbols that cause the drugs influence the beliefs and attitudes as well as the meanings given to the experiences of drug taking. ${ }^{15}$ The shape, color and size of a tablet can influence the placebo effect. The very small pills have the meaning of being very powerful, and the very large ones also, as opposed to the medium ones. There may be a different response depending on the color of the pills, especially in patients with mental health problems: anxiety responds more quickly to green, while depression does to yellow. The more pills that are prescribed the higher the result (three placebo tablets get better results than the one of the active drug $)^{7}$ On the other hand, price also influences nocebo effects; ${ }^{9}$ for example, it has been reported that placebo medication for Parkinson's disease was more effective when it was perceived as expensive. ${ }^{16}$

\section{The situation and the way in which the placebo is prescribed: the doctor-patient relationship}

The profound effect of the doctor-patient relationship in the treatment is known. It can be differentiated two different effects that may overlap in part:

\section{The doctor himself as a drug}

The relationship of trust may be sufficient to improve symptoms of stress or anxiety, so that the effect attributed to any prescribed medication may be due to that relationship. It is about "the effect of the doctor himself as a drug". The medicine most frequently used by general practitioners is the doctor himself. Consequently, the doctor himself should be considered as a drug, that is, that the concepts of 
pharmacology, such as overdoses, allergic reactions, side effects, etc., can be applied to the interaction between doctor and patient. Both, doctor and patient, are modified: one towards the other and vice versa. ${ }^{17-20}$ Studies that have used functional magnetic resonance imaging show that there are areas of the brain that are activated after taking a sugar pill if a doctor tells you that what you are taking is a medication. ${ }^{3}$ Therefore, there is a "miraculous moment" when the mere presence of the doctor is the most effective part of the treatment. In practice, many patients are helped by the doctor for different reasons; a main one is that the cure is produced by the mere presence of the doctor and the confidence generated-placebo effect-plus certain factors of the patient himself (desire or desire to live, beneficial role of positive emotions and cooperative work doctor-patient). In any case, of course, obviously the doctor must make a correct diagnosis to determine if specific treatment is necessary. In this way, it can be said that the placebo is not a pill, it is a process. A process that begins with the patient's confidence in the doctor and it extends through full immunological functioning and the "healing system" of the patient. The placebo is the proof that there is no true separation between mind and body. Illness is always an interaction between both; it can start in the mind and affect the body, or start in the body and affect the mind. Attempts to treat mental illnesses as completely free of physical causes and to treat organic diseases as if they had no relation to the mind, is somewhat archaic. The chances of success in a treatment are directly proportional to the quality of the doctor-patient relationship. In summary, in this sense, the doctor is in itself the most powerful placebo, and there are numerous experimental studies that demonstrate it. The history of medicine is the history of the placebo effect. ${ }^{1}$

\section{The effect of the physician's form and attitudes on the drug in question}

Even when there is a true pharmacological effect of the prescribed active drug, it should be expected that its effect will be modified considerably by the optimism or confidence expressed by the doctor before the treatment. The results of the treatments are more dependent on the personality of the therapist than on the pharmacological effect or the technique used. There is evidence that health professionals can influence patients about the way they think and feel about their illnesses or their treatment. Therefore, the "how" of prescribing is than important as what it is prescribed ${ }^{21}$ Not surprisingly, a pharmacological prescription whose decision is expressed by the doctor with great security, generally has a placebo effect; On the other hand, if the decision is considered to have a dubious effect, it will often give rise, depending on the general context of the consultation and the personality characteristics of the patient, to a nocebo effect, with deficient therapeutic results or adverse pharmacological results. And we must remember that these effects are not mediated by the drug, but psychosocially. ${ }^{7-25}$ It has also been shown that the nocebo effect plays a role in introducing a new drug or in changing an established medicine, for example, by switching patients from a reference biological product to a biosimilar, which has repercussions on both the medical- patient relationship and in the healthcare costs. ${ }^{8,9}$ Here, also it should be included "the effect of knowing the diagnosis", which can lead to a nocebo effect: when a patient is told that he is hypertensive, for example, the GP observes that it is very common that patient, until that Asymptomatic moment, report presenting a series of symptoms such as headache, dizziness, asthenia, palpitations, etc. In this same sense, another example of nocebo effect would be the frank revelations of the GP on the imminence of a degenerative process of the patient that trigger and accelerate said process. ${ }^{26,27}$

\section{The psychosocial factors of the patient receiving the placebo}

Likewise, patients can also influence the response of the drug. The expectations that the patient has about the prescribed drug affects the outcome of the treatment. Although the placebo effect of the doctorpatient relationship rests mainly with the physician, some patients seem to react more likely to that effect than others. However, although predictive factors have been proposed in some diseases, ${ }^{28}$ in practice it is difficult to completely predict which patients will respond to placebo or nocebo. ${ }^{11}$ Although the predictors of the placebo response have yet to be discovered in a safe and operational way, it has been suggested that the response probably has more than one mediator, and that consequently the interaction of several different mediating factors, in the general medicine, is probably responsible for the placebo response..$^{29}$ In any case, several strategies have been proposed to maximize the placebo effect and minimize the nocebo effect. That is, to be able to know, at least in part, patients reactors or responders to the placebo phenomenon, and with the limitations mentioned above, it seems that these responders are preferably young patients, and of low intelligence level, possibly more women than men (men report fewer adverse effects than women), with a high level of sociability and desire to please..$^{7-30}$

In general medicine, where the GP "plays with advantage", because in his patient's continuous attention he knows the personality traits of these and he can know with great approximation, in a given patient, if he is an anxious person (people who perceive the situations such as dangerous or threatening, or cautious, tense, easily fatiguing, timid, apprehensive, pessimistic, etc.); or if the patient is a person with a depressive personality (individuals who are characterized by being particularly serious, unable to have fun or relax and without humor, with negative, pessimistic thoughts, etc., even without there being a mental illness identifiable as anxiety and / or depression). All these characteristics increase the probability of nocebos effects. And consequently the GP could predict the response for a given patient of the prescription of a drug with utility of the same and improvement or intolerance to the drug. This cataloging of patients, which is typical of the characteristics of continued relationship, is of great importance for the taking of therapeutic decisions. ${ }^{24-32}$

\section{The disease to be treated}

Numerous psychosocial factors affect the diseases themselves, modifying experiences and emotions, and consequently modify the doctor-patient relationship and the placebo effect. These psychosocial factors of diseases include, among others, feelings of anguish, guilt, punishment, fear of death, stress, low self-esteem, worthlessness, denial, anger, frustration, irritability, impatience, altered body image, discrimination, stigma, shame, dependence, vulnerability, disability, social isolation, changes in habits, social maladjustment, work, school and economic problems, etc. And different types of them are present in diseases such as, cardiovascular diseases, bronchial asthma, COPD, rheumatoid arthritis, celiac disease, digestive functional disorders, intestinal inflammatory diseases, gastroesophageal reflux, migraine, stroke, multiple sclerosis, epilepsy, neurodegenerative diseases, Parkinson's , psychiatric diseases, diabetes mellitus, hyperthyroidism, obesity, sexual disorders, AIDS, hepatitis C, cancer, etc. ${ }^{33}$ In addition, the representations of health and disease are dependent on the cultural context, even though there are points in common, there are perceptions of different psychosocial aspects according to cultures: it 
is said that in Eastern Europe they focus on the "internal strengths", the South Americans in the "love" and the Italians in the "personal autonomy", etc. ${ }^{34}$ All this makes it very difficult to have a complete list of the effectiveness of placebo for all important diseases, which is still lacking. ${ }^{11}$ In any case, it has been reported that the effect of placebos is more intense in acute stressful situations, such as postsurgical pain, headache or anxiety. ${ }^{7}$

\section{The mode of action of the placebo}

The placebo does not act purely by suggestion. The placebo effect is not only a psychological effect or something that depends solely on our attitude or our perception. Placebo and nocebo effects are psychobiological events imputable to the therapeutic context. Their major mechanisms are expectancy and classical conditioning. ${ }^{6}$ Placebo and nocebo represent complex and distinct psycho neurobiological phenomena in which behavioural and neurophysiologic modifications occur together with the application of a treatment..$^{35}$ Placebonocebo responses and the neuro-bio-behavioural mechanisms underlying the placebo and nocebo responses that are currently known in terms of different diseases and physiological systems are mediated through changes in various cortico-subcortical networks and psychophysiological systems and have an impact on molecular level. ${ }^{4-38}$ Nocebo and placebo effects engage a complex set of neural circuits in the central nervous system that modulates the perception of touch, pressure, pain, and temperature. ${ }^{9}$ The recent research has revealed that these placebo-induced biochemical and cellular changes in a patient's brain are very similar to those induced by drugs. ${ }^{5}$ Placebo effects rely on complex neurobiologic mechanisms involving neurotransmitters (eg, endorphins, cannabinoids, and dopamine) and activation of specific, quantifiable, and relevant areas of the brain (eg, prefrontal cortex, anterior insula, rostral anterior cingulate cortex, and amygdala in placebo analgesia). ${ }^{39}$ It has also been reported that these changes seem to depend on a family of neurotransmitters called catecholamines - to which adrenaline, noradrenaline and dopamine belong - implicated among other factors in the stress response, and certain mutations have been discovered in a an enzyme called catecholmethyltransferase (which alter the levels of these neurotransmitters) that can predict whether a patient will have a greater or lesser placebo effect, demonstrating that this effect may have a genetic basis. ${ }^{3}$

\section{The side effects of placebo (nocebo)}

Numerous subjective adverse effects, such as drowsiness, dry mouth, headache, nausea, etc., are reported in many placebo studies, including serious adverse reactions such as angioneurotic edema, as well as dependence on placebo. ${ }^{7}$

\section{The use of placebo and its ethical aspects}

Given the medically proven fact that placebo treatments work, doctors could include "fake treatments" in their medical repertoire. However, this poses an ethical dilemma. ${ }^{40}$ The doctor could give the placebo with the intention of improving the patient's situation, and in the knowledge that it probably will not make it worse, but this indication is ill-indicated given the influences of numerous psychosocial factors, in addition to ethical issues. It is usual for the doctor to prescribe drugs of doubtful efficacy, whose effect is mainly due to the placebo effect, in the error that it is due to the pharmacological action, or as a way to reassure the doctor and the patient when the doctor is doing what is expected to do: prescribe a drug at the end of the consultation. ${ }^{7-20}$
For daily practical therapeutic purposes, placebos may be ethically acceptable and lawful if there is no effective therapy available, if the complaints are minor, if the patient expressly wishes treatment, and if there is a reasonable likelihood of success. However, an explanation of the expected benefits and risks must be provided to the patients. ${ }^{11}$

\section{Interaction of specific and non-specific effects}

Conventional medical research usually assumes that, on one side, the specific or pharmacological effects of medical and, on the other hand, the psychosocial or nonspecific interventions are simply additive and do not interact, but this assumption has never been demonstrated. ${ }^{25-33}$ That is to say, it is assumed that the components of an integrative, biopsychosocial or person-centered intervention have an additive relationship to produce the total effect. This model assumes an additive relationship between placebo and the effects of active drugs and, therefore, presents an arithmetic sum. However, this additive model can be questioned. It could be say that In a statistical model the combined effect of several factors is the sum of the effects produced by each of the factors in the absence of others (for example, if a factor increases the risk in $\mathrm{a} \%$ and a second factor in a $\mathrm{b} \%$, then the additive combined effect of the two factors is $(a+b) \%)$. But, evidence has shown that the placebo effect can influence the clinical effect of the active ingredient. And other researchers have suggested that specific as well as non-specific effects are sometimes synergistic, and others antagonistic. In consequence, the researchers conclude that the implicit additive model is therefore too simplistic: and there are numerous effects that interact. ${ }^{41}$

\section{Does the placebo cause healing or relief of symptoms?}

Although the placebo effect can provide relief, they rarely provide heal. Although research has revealed objective neurobiological pathways and correlations of placebo responses, evidence to date suggests that the therapeutic benefits associated with placebo effects probably do not alter the pathophysiology of diseases beyond their symptomatic manifestations; that is, they mainly involve subjective and self-evaluated symptoms. For example, there is no evidence that the placebo effect can reduce tumors; however, experiments show that common cancer symptoms and side effects of cancer treatment (e.g., fatigue, nausea, hot flushes and pain) respond to placebo treatments. Similarly, an experiment in patients with asthma showed that placebos do not affect the forced expiratory volume of patients in 1 second, but still can dramatically relieve perceived symptoms. In any case, the effects of the symbols and the clinical interactions that produce the phenomenon of placebo can dramatically improve the effectiveness of pharmaceutical products, ${ }^{39}$ and it can be thought that a placebo, intermittently paired with a dose of therapeutic drug, it could produce continuous therapeutic effects with fewer side effects. ${ }^{42}$

\section{Conclusion}

The effects of placebo and nocebo occur frequently and are clinically significant, but are not recognized in clinical practice. The psychobiological effects of the context exist, even in the clinical setting, even if it has not been applied consciously. The phenomenon of placebo / nocebo does not imply a new theoretical scenario in the biopsychosocial conceptual framework of general medicine, but it forces us to think about aspects that usually can not be taken into account, and may have profound implications for medical practice in general medicine, as well of for clinical trials. GPs should be able to recognize these phenomena and master tactics on how to 
manage these effects to improve the quality of clinical practice. The characteristics of the GP, the characteristics of the patient, the doctor-patient relationship, the characteristics of the treatment and the disease, and the general medical care environment are, among others, contextual factors that influence the clinical results. Patient expectations are important determinants of unwanted adverse effects, and GPs must adopt a strategy that encourages optimal treatment outcomes. Each treatment in general medicine determines certain biological, psychological and social effects; these can be specific and contextual or nonspecific effects, which interact with each other. GPs must manage the contextual factors of the placebo effect as a driver of any active therapeutic intervention to improve the positive effects of the treatment and avoid harmful nocebo effects. Consequently, GPs must combine active medication with a specific context and an adequate level of therapeutic contact, to improve non-specific effects of treatment and obtain a greater response to treatment. Since a placebo effect can be produced in any standard therapy, information about the placebo effect should be provided during medical education and continuing medical education.

\section{Acknowledgments}

None.

\section{Conflicts of interest}

The author declares there is no conflicts of interest.

\section{References}

1. Cousins N. Anatomy of an illness as perceived by the patient. Reflections on healing and regeneration. New York: W.W. Norton \& Company. 1979.

2. Haygarth J. Of the imagination, as a cause and as a cure of disorders of the body: exemplified by fictitious tractors, and epidemical convulsions. Read to the Literary and Philosophical Society of Bath. Literary and Philosophical Society of Bath; University of Leeds. Publisher Bath: Printed by R. Cruttwell, and sold by Cadell and Davies, Strand, London Contributor University of Leeds Library: Digitizing sponsor Jisc and Wellcome Library. 1801.

3. Mulet JM. ¿Y si el efecto placebo fuera algo más que un truco de la mente? El País. 2018.

4. Jakovljevic M. The placebo-nocebo response: controversies and challenges from clinical and research perspective. Eur Neuropsychopharmacol. 2013.24(3):333-341.

5. Benedetti F, Amanzio M. The placebo response: how words and rituals change the patient's brain. Patient Educ Couns. 2011;84(3):413-419.

6. Chavarria V, Vian J, Pereira C, et al. The Placebo and Nocebo Phenomena: Their Clinical Management and Impact on Treatment Outcomes. Clin Ther. 2017;39(3):477-486.

7. Silverstone T, Turner P. Drug treatment in psychiatry. London: Routledge \& Kegan Paul Ltd. 1974

8. Kristensen LE, Alten R, Puig L, et al. Non-pharmacological Effects in Switching Medication: The Nocebo Effect in Switching from Originator to Biosimilar Agent. BioDrugs. 2018;32(5):397-404.

9. Colloca L. Nocebo effects can make you feel pain. Science. 2017;358(6359):44.

10. Colloca L, Miller FG. Role of expectations in health. Curr Opin Psychiatry. 2011;24(2):149-155.
11. Scriba PC. Placebo and the relationship between doctors and patients. Overview. Bundesgesundheitsblatt Gesundheitsforschung Gesundheitsschutz. 2012;55(9):1113-1117.

12. Turabian JL, Perez Franco B. Prescription of medicines in family medicine: rational, reasonable or relevant?. Aten primaria. 2005;36(9):507-509.

13. Turabian JL. Biopsychosocial Concept is the Characteristic that Distinguishes the Practice of General Practitioner/Family Doctor. J Gen Pract (Los Angel). 2018;6:e122.

14. Benedetti F. L'effeto placebo. Breve viaggio tra mente e Corpo. Roma: Carocci editore. 2018

15. Turabian JL, Perez franco B. [Non-pharmacological aspects of medications. Semergen. 2011;37(5):246-251.

16. Espay AJ, Norris MM, Eliassen JC, et al. Placebo effect of medication cost in Parkinson disease: a randomized double-blind study. Neurology. 2015;84(8):794-802.

17. Balint E, Norell JS. Six minutes for the patient: interactions in general practice consultations. London: Tavistock Publications. 1973.

18. Balint M, Hunt J, Joyce D. Treatment or diagnosis. A study of repeat prescriptions in general practice. London: Tavistock Publications. 1984.

19. Turabián Fernández JL, Pérez Franco B. The concept of treatment in familiy medicine: A contextualised and contextual map of a city hardly seen. Aten Primaria. 2010;42(5):253-254.

20. Turabian JL. Doctor-Patient Relationship in Pharmacological Treatment: Discontinuation and Adherence. COJ Rev \& Res. 2018;1(5).

21. Luban-Plozza B. Psychological aspects of drugs. Soz Praventivmed. 1980;25(1-2):56-60.

22. Faasse K, Petrie KJ. The nocebo effect: patient expectations and medication side effects. Postgrad Med J. 2013;89:540-546.

23. Evers AWM, Colloca L, Blease C, et al. Implications of Placebo and Nocebo Effects for Clinical Practice: Expert Consensus. Psychother Psychosom. 2018;87(4):204-210.

24. Turabian JL, Moreno-Ruiz S. The fable of the pine and the palm tree: the two extremes. Strategies to maximize the placebo effect and minimize the nocebo effect in primary health care. Ment Health Addict Res. 2016;1(3):44-46.

25. Turabian JL. Pharmacological Non-Prescription, Doctor-Patient Relationship and Biopsychosocial Approach: Foreign Lands or Foreign Travelers? Archives of Community and Family Medicine. 2018;1(2):39_ 42.

26. Punset E. Cara a cara con la vida, la mente y el universo. Conversaciones con los grandes científicos de nuestro tiempo. Barcelona: Destino. 2007.

27. Colloca L, Finniss D. Nocebo Effects, Patient-Clinician Communication, and Therapeutic Outcomes. JAMA. 2012;307:567-568.

28. King BH, Dukes K, Donnelly CL, et al. Baseline Factors Predicting Placebo Response to Treatment in Children and Adolescents With Autism Spectrum Disorders: A Multisite Randomized Clinical Trial. JAMA Pediatr. 2013;167(11):1045-1052.

29. Weimer K, Colloca L, Enck P. Placebo effects in psychiatry: mediators and moderators. Lancet Psychiatry. 2015;2(3):246-257.

30. Bingel U, Placebo Competence Team. Avoiding nocebo effects to optimize treatment outcome. JAMA. 2014;312(7):693-694.

31. Clark LA, Watson D, Mineka S. Temperament, personality, and the mood and anxiety disorders. J Abnorm Psychol. 1994;103(1):103-116. 
32. Gutiérrez-Islas, Báez-Montiel BB, Turabian JL, Patients with adverse drug reactions have a higher prevalence of emotional disorders. Aten Primaria. 2012;44:720-726.

33. Turabián JL, Pérez Franco B. Journey to what is essentially invisible: Pysochosocial aspects of disease. Semergen. 2014;40(2):65-72.

34. Pace CS, Velotti P, Zavattini GC. Representations of health and illness by Eastern European, South American and Italian care workers: a qualitative study. J Health Psychol. 2012;17:490-499.

35. Testa M, Rossettini G. Enhance placebo, avoid nocebo: How contextua factors affect physiotherapy outcomes. Man Ther. 20165;24: 65-74.

36. Schedlowski M, Enck P, Rief W, et al. Neuro-Bio-Behavioral Mechanisms of Placebo and Nocebo Responses: Implications for Clinical Trials and Clinical Practice. Pharmacol Rev. 2015;67(3):697730.

37. Bartoli F, Clerici M, Di Brita C, et al. Effect of clinical response to active drugs and placebo on antipsychotics and mood stabilizers relative efficacy for bipolar depression and mania: A meta-regression analysis. $J$ Psychopharmacol. 2018;32(4):416-422.

38. Hamblin J. Placebo Effect of the Heart. The Atlantic. 2017;8.

39. Kaptchuk TJ, Miller FG. Placebo Effects in Medicine. $N$ Engl J Med. 2015;373:8-9.

40. van Deventer MO. Meta-placebo: do doctors have to lie about giving a fake treatment? Med Hypotheses. 2008;71(3):335-339.

41. Boehm K, Berger B, Weger U, et al. Does the model of additive effect in placebo research still hold true? A narrative review. JRSM Open. 2017;8(3):2054270416681434.

42. Albring A, Wendt L, Benson S, et al. Preserving learned immunosuppressive placebo response: perspectives for clinical application. Clin Pharmacol Ther. 2014;96(2):247-255. 